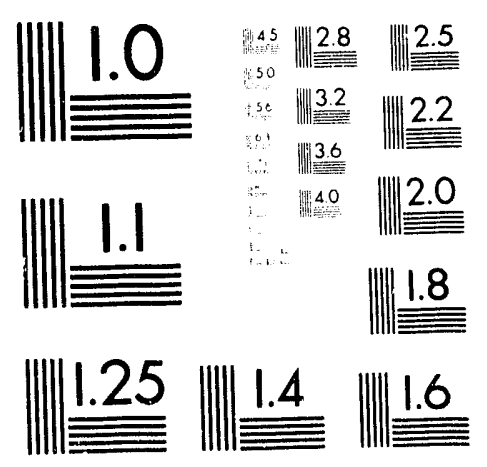



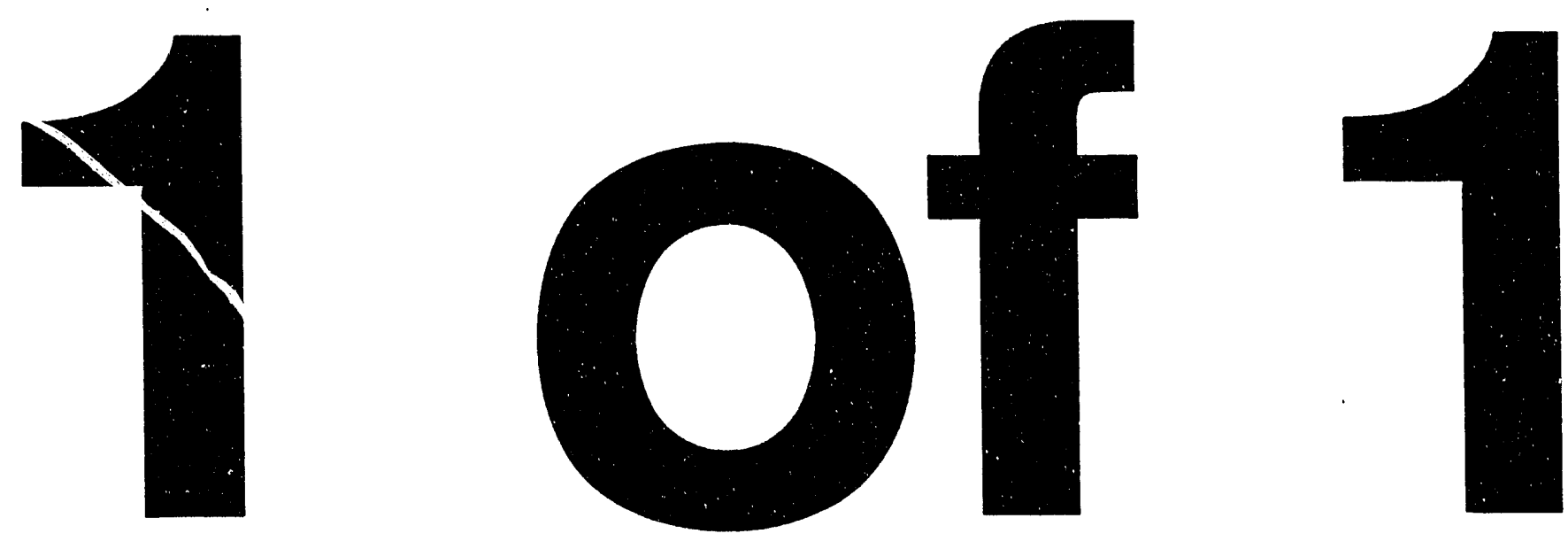


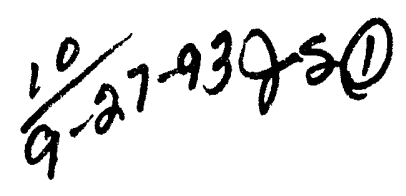

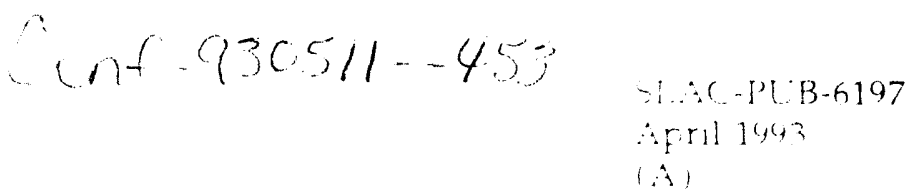

\title{
Localized Chromaticity Correction of Low-Beta Insertions in Storage Rings*
}

\author{
M. Donald, R. Helm, J. Irwin, H. Moshammer \\ Stanford Linear Accelerator Center, Stanford, CA 94309 \\ E. Forest, D. Robin, A. Zholents \\ Lawrence Berkeley Laboratory, Berkeley, CA 94720 \\ M. Sullivan \\ University of California Intercampus Institute for Research at Particle Accelerators, \\ Stanford Linear Accelerator Center, Stanford, CA 94309
}

\begin{abstract}
The correction of the chronaticity of low-beta insertions in the storage rings is usually made with sextupole lenses in the ring's arcs. When decreasing the beta functions at the insertion point (IP), this technique becomes fairly ineffective, since it fails to properly correct the higher order chromatic aberrations. Here we consider the approach where the chromatic effects of the quadrupole lenses generating low beta functions at the IP a re corrected locally with two families of sextupoles, one family for each plane. Each family has two pairs of sextupoles which are located symmetrically on both sides of the IP. The sextupole-like aberrations of individual sextupoles are eliminated by utilizing optics forming a $-i$ transformation between sextupoles in the pair. The optics also includes bending magnets which preserve equal dispersion functions at the two sextupoles in each pair. At sextupoles in one family, the vertical beta function is made large and the horizontal is made small. The situation is reversed in the sextupoles of the other family. The betatron phase advances from the IP to the sextupoles are chosen to eliminate a second order chromatic aberration. The application of the localized chromatic correction is demonstrated using as an example the lattice design for the Low Energy Ring of the SLAC/LBL/LLNL PEP-II B Factory.
\end{abstract}

\section{INTRODUCTION}

Chromatic correction is a common requirement in many optical and electronic systems as well as charged particle beam lines. It has been noted in several contexts that local chromatic correction is preferable. In storage rings, it is usual to correct chromatic aberrations with sextupole families in the FODO arrays of the arcs. For colliding rings with very small $\beta$-function values at the interaction point (IP), large values of chromaticity are generated in the final focusing quadrupoles. Attempts to correct this with arc sextupole families can fail for two reasons: i) the transport of chromaticity through a lattice gives rise to higher order chromaticity which remains uncompensated, and ii) the arc sextupoles, which typically are interleaved, create unacceptable amounts of octupole aberration when they are strengthed to compensate for additional chromaticity.

This paper describes the adaptation of a scheme used in the final focus systems of linear colliders to locally compensate chromatic abcrrations of final

* Work supported by Department of Energy contracts DE-AC03-76SF00515, DE-AC03-76SF00098, and DEASO3-76ER70285.

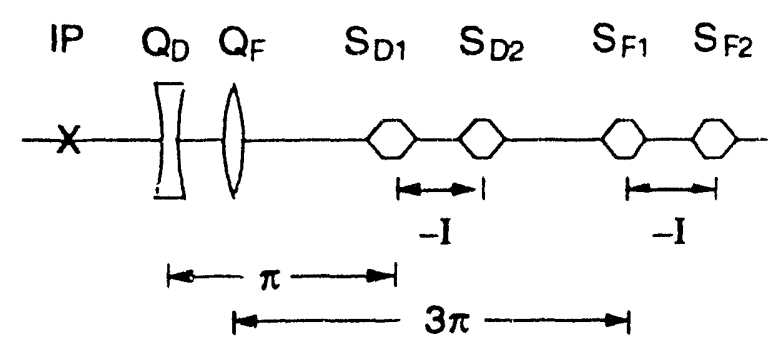

Figure 1. A schematic for a typical final focus chromatic correction system

doublets [Ref 1]. It consists of two pairs of noninterleaved sextupoles in the interaction region (IR) on either side of the IP.

\section{A. Description of Typical Chromaticity Correction Scheme}

The typical final focus chromatic correction system, shown in Fig. 1, consists of two pairs of sextupoles. Each sextupole of a pair is placed -I from the another, so that the sextupole aberration is cancelled, and at $n \pi$ phase difference from the quadrupole whose chromaticity is being compensated. Typically the vertical plane is more strongly focused than the horizontal, so the vertical compensation is closest to the IP. At this pair of sextupoles, the vertical $\beta$-function is large, and the horizontal $\beta$-function is small. The linear transformer between the first and second pairs of sextupoles reverses this $\beta$-function ratio so that the second sextupole pair primarily compensates the horizontal chromaticity of the final doublet. Beyond the second sextupole pair is a $\beta$-matching section. From the begining of the $\beta$-match through the IP, the system has only a small residual chromatically, and so is chromatically transparent when placed in a storage ring. Of course, in a storage ring there needs to be an identical system on the other side of the IP.

The dispersion function is an important feature of the compensation scheme described above. There must be dispersion it the sextupoles, usually equal at both sextupoles of a pair, so that the sextupole strengths are at a minimum, and certain other aberrations, such as high-order dispersion arising in the -I section, are cancelled. Typically there is a bend in the $\beta$-match section $\pi / 2$ from the first sextupole, to launch a dispersion function, a bend at the middle of each -1 to symmetrize the dispersion function, and a bend in the final telescope $\pi / 2$ from the final sextupole to terminate the dispersion.

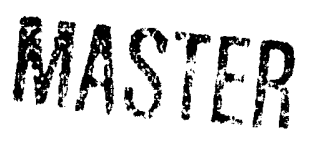

Presented at the Particle Accelerator Conference (PAC 93), Washington. DC, May 17-20, 1993 


\section{Hinl}

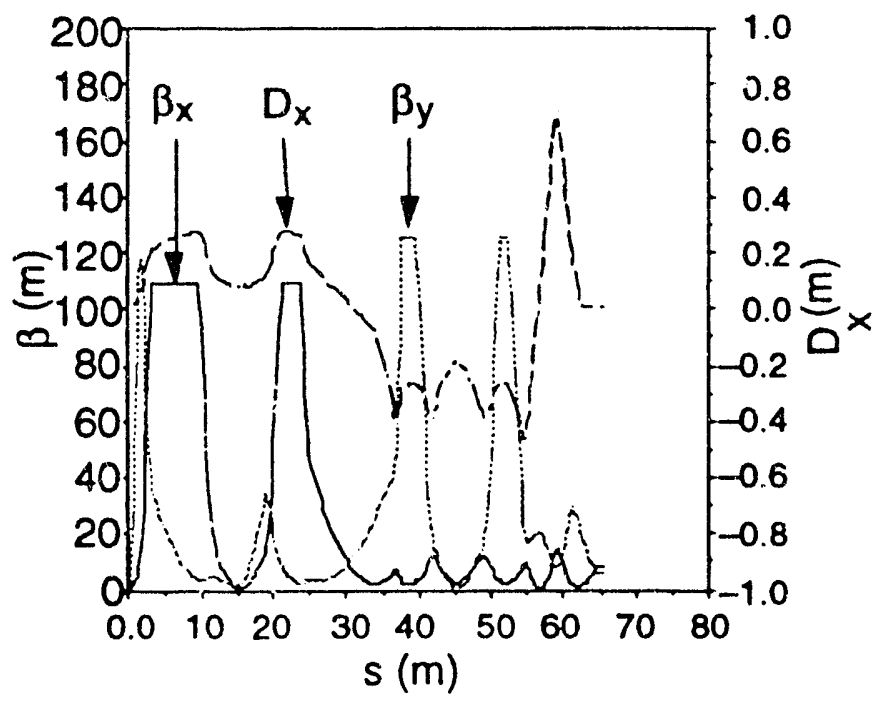

Figure 2. The lattice functions for the LER IR chromatic correction system.

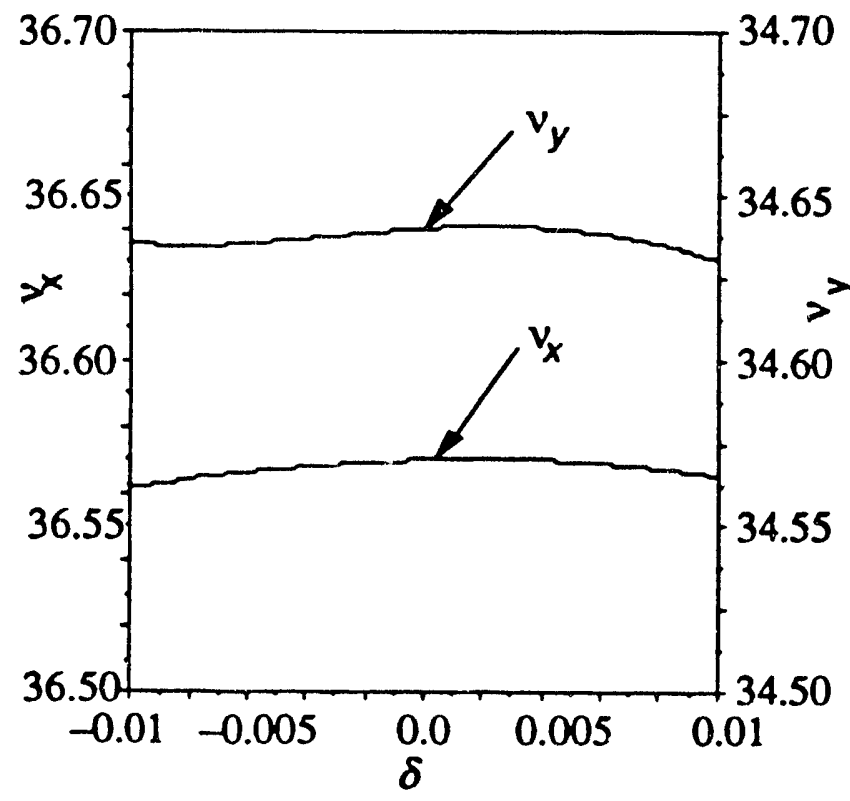

Figure 3. The horizontal and vertical tune shift of the LER as a function of energy.

\section{B. Compensation of Second-Order Chromatic Terms}

The second-order chromatic terms in the one-turn nonlinear generator arise from the first Poisson bracket $1 / 2\left[\mathrm{H}_{\mathrm{i}}, \mathrm{H}_{\mathrm{j}}\right]$ in the $\mathrm{CBH}$ theorem expansion, where $\mathrm{H}_{\mathrm{i}}$ is the chromatic-aberration Hamiltonian coming from a quadrupole or from a sextupole at a location with horizontal dispersion. For a quadrupole, $\mathrm{H}_{\mathrm{i}}=-1 / 2 \mathrm{kQi} \delta$ $\left(x_{i} 2-y_{i} 2\right)$. For a sextupole, $k_{Q i}$ is replaced by $k_{S i} \eta_{i}$. In other words, the higher-order chromatic term arises from chromaticity in one quadrupole (or sextupole) modifying the chromatic contribution of another

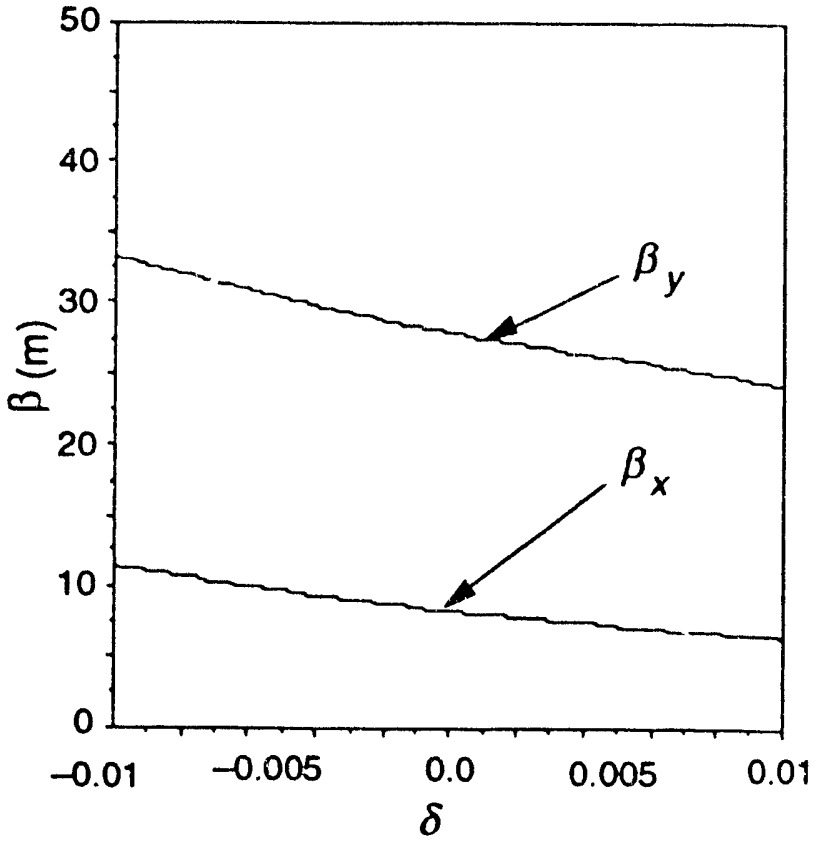

Figure 4. The horizontal and vertical $\beta$-function at the $R F$ cavity in the LER as a function of energy.

quadrupole (or sextupole). This Poisson bracket can be evaluated to yield $1 / 2\left[\mathrm{H}_{j}, \mathrm{H}_{j}\right]=1 / 2 \mathrm{kQi} \mathrm{kQj}_{\mathrm{j}} \delta^{2}\left(\left[\mathrm{x}_{\mathrm{i}}, \mathrm{x}_{\mathrm{j}}\right] \mathrm{x}_{\mathrm{i}} \mathrm{x}_{\mathrm{j}}\right.$ $\left.+\left[y_{i}, y_{j}\right] y_{i} y_{j}\right)$. The factor $\left[x_{i}, x_{j}\right]\left(\left[y_{i}, y_{j}\right]\right)$ is just the $(1,2)$ $[(3,4)]$ element of the linear transfer matrix between quadrupoles $i$ and $j$. If these elements are separated by $n \pi$, their interaction is zero, and away from $n \pi$ this term can take one sign or the other. This feature of the source of second-order terms can be used to advantage within the framework of the chromaticity compensation scheme we have described above [Ref 4]. By slightly varying the phase shift between the sextupole pair and the chromatic source, it is possible to vary the sign of the second-order chromatic term and compensate, for example, any second-order tune shift term that remains in the full ring.

\section{Description of the PEP-II LER Chromaticity Correction} Scheme

The scheme described above was studied for PEP-II LER [Ref 3]. This effort was unsuccessful because space constraints required strong intermediate quadrupoles which generated unacceptable chromaticty between sextupole pairs. A solution for the PEP-II low energy ring was achieved by placing a sextupole at the final doublet. As a result of this bend and an offsetquadrupole [Ref 4] near the IP, large dispersion and a large horizontal $\beta$-function exist at the $F$ quadrupole of the doublet. By beginning compensation immediately, additional space becomes available, and the strong quadrupole problem mentioned above is resolved. Figure 2 shows the lattice functions for the completed design. The sextupole near the first $F$ quadrupole is placed where $\beta_{y}$ is small. The interchange of the vertical and horizontal compensation, with the horizontal compenstions closest to the IP, is acceptable because the 
siluation is less extreme than in the FFTB where $\beta_{\mathrm{y}}{ }^{*}=0.1 \mathrm{~mm}$.

Because the correction is local, precisely $n \pi$ ( $n=0$ or $\mathrm{n}=2$ ) from the source, both the tune-shift with energy and variation of the $\beta$-function, with energy $(d \beta / d \delta)$ are compensated. Since uncompensated first-order terms give rise to higher-order terms, we have also reduced higher-order chromatic terms. Figure 3 shows the tuneshift as a function of energy. Little first- or second-order dependence on $\delta$ remains in the PEP-II LER lattice. Figure 4 shows $\beta_{x}(\delta)$ and $\beta_{y}(\delta)$ at the RF cavity, where a $\delta$-dependent $\beta$-function could drive synchro-betatron resonances. $\beta_{x}(\delta)$ and $\beta_{y}(\delta)$ are well behaved here and at other locations throughout the ring.

A local chromatic correction scheme with sextupoles near the IP was previously used in VEPP-4 [Ref 3]. In this case there was dispersion at the final doublet due to the choice of a vertical solenoidal magnetic field. However, in VEPP-4 there were only one SD and one SF on cach side of the IP. A scheme similar to VEPP-4 was also considered for designs of storage rings with monochromator optics [Ref ó].

\section{REFERENCES}

[1] Oide, SLAC-PUB-4953, 1989.

[2] Private Comm. K. Brown and R. Helm compensated 2nd-order chromaticty in their FFIB designs.

[3] E. Forest, et al, LBL-33479, Jan 1993. Submitted to KEK B-factory workshop.

[4] PEP-II B-factory CDR.

[5] VEP -4 report on All Union Conf. for Charged Particle Accelerators.

[6] A. Zholentz, CERN-CL-92-27(AP)

\title{
DISCLAIMER
}

\begin{abstract}
This report was prepared as an account of work sponsored by an agoncy of the United States Government. Neither the United States Government nor any agency thereof, nor any of their employees, makes any warranty, express or implied, or assumes any legal liability or responsibility for the accuracy, completeness, or usefulness of any information, apparatus, product, or process disclosed, or represents that its use would not infringe privately owned rights. Reference herein to any specific commercial product, process, or service by trade name, trademark, manufacturer, or otherwise does not necessarily constitute or imply its endorsement, recommendation, or favoring by the United States Fovernment or any agency thereof. The views and opinions of authors expressed herein do not necessarily state or reflect those of the United States Government or any agency thereof.
\end{abstract}



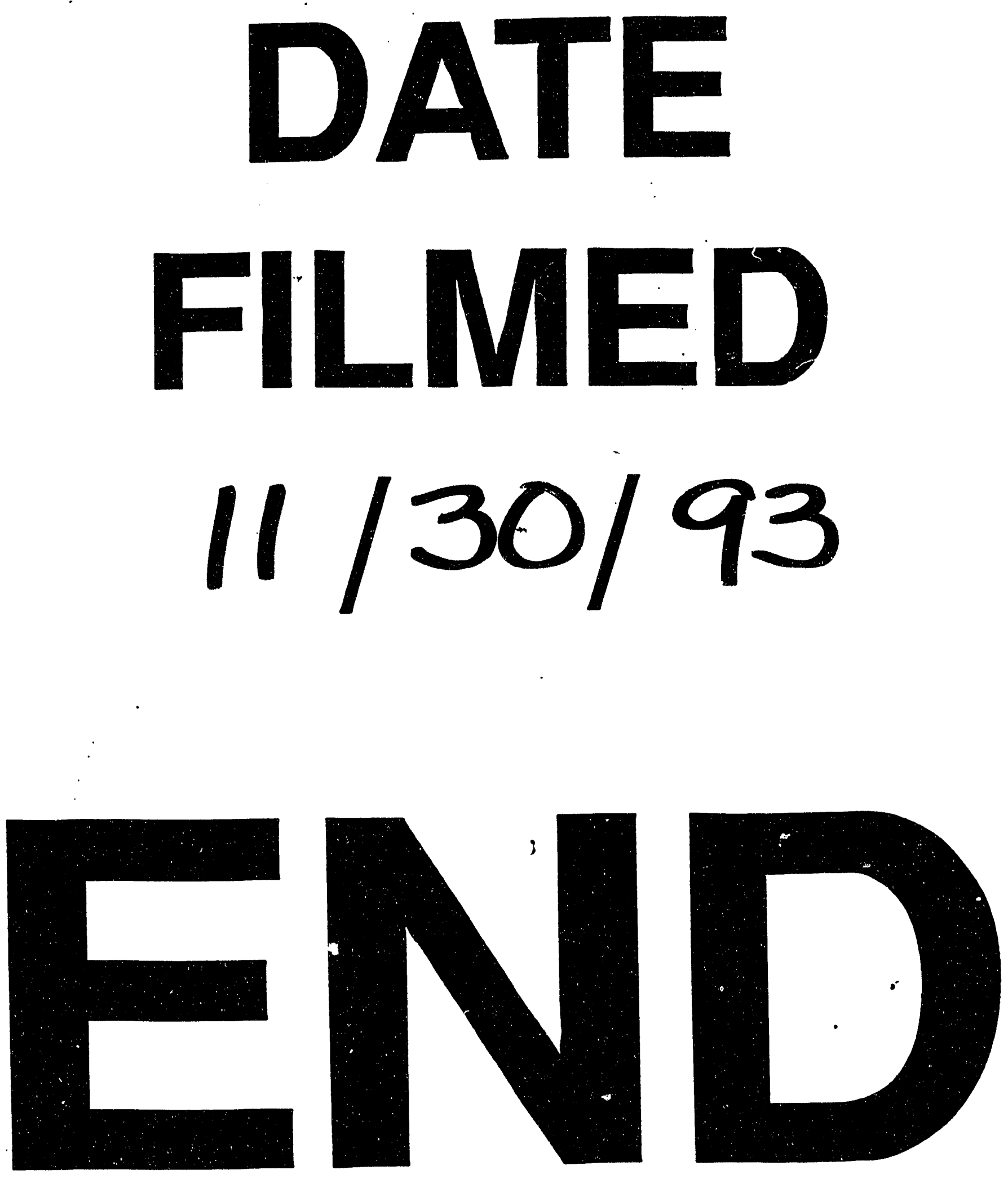
